\title{
Studying strictly positive secure capacity in cognitive radio-based non-orthogonal multiple access
}

\author{
Chi-Bao Le, Dinh-Thuan Do \\ Faculty of Electronics Technology, Industrial University of Ho Chi Minh City (IUH), Ho Chi Minh City, Vietnam
}

\begin{abstract}
Article Info
Article history:

Received Oct 16, 2020

Revised Jan 7, 2021

Accepted Feb 16, 2021

\section{Keywords:}

Cognitive radio

NOMA

Strictly positive secure capacity

ABSTRACT

This paper studies a downlink security-aware secure outage performance in the secondary network of cognitive radio-assisted non-orthogonal multiple access network (CR-NOMA). The multiple relay is employed to assist transmission from the secondary source to destinations. The security-aware performance is subject to constraints in fixed power allocation factor assigned to each secondary user. The security-aware secure performance is based on channel state information (CSI) at the physical layer in which an eavesdropper intends to steal information. According to the considered system, exact expressions of Strictly positive secure capacity (SPSC) are proved to analyze system in terms of secure performance. Finally, the secondary user secure problem is evaluated via Monte-Carlo simulation method. The main results indicate that the secure performance of proposed system can be improved significantly.
\end{abstract}

This is an open access article under the CC BY-SA license.

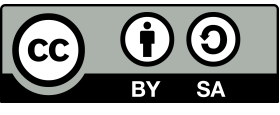

\section{Corresponding Author:}

Dinh-Thuan Do

Faculty of Electronics Technology, Industrial University of Ho Chi Minh City

12 Nguyen Van Bao Road, Go Vap District, Ho Chi Minh City, Vietnam

Email: dodinhthuan@iuh.edu.vn

\section{INTRODUCTION}

Both non-orthogonal multiple access (NOMA) and physical-layer security (PLS) have been introduced as promising enabling technologies to implement some applications for Internet of Things (IoT) or future systems [1-4]. Recently, the coexistence of these two important communication techniques benefits from cognitive ability and massive connections and these hybrid techniques have been considered to provide spectral efficient improvement for wireless transmission such as recent work [6-8].

Furthermore, physical-layer security (PHY) has attracted great interests while wireless applications are more popular. PHY based secure method does not require extra resources for the secret key [10-14]. In order to achieve secure communication, one can exploit the physical layer characteristics of the wireless channels. However, the secrecy rate of wireless communication systems is constrained by the channel state information [15]. In order to improve the secrecy rate, many methods are introduced such as jamming, multiple antennas, cooperative relaying and artificial noise (AN)-aided techniques have been studied [16]. Main results reported in [17-24] that these techniques benefit to improve the secrecy rate.

Interestingly, most existing works have mainly focused on the performance and optimization of the PLS in NOMA systems . However, there is still open problems to rigorously study the feasibility of achieving the better secure performance by using best relay selection in secondary network of CR-NOMA systems. Although the joint user scheduling and power allocation problems are investigated for NOMA-based wireless network in [17], how cognitive radio technology affects the secure performance for NOMA-based wireless network needs further studies. In this paper, we study the security-aware SPSC metric CR-NOMA network. 


\section{SYSTEM MODEL}

In Figure 1, we consider the CR system containing the secondary network including base station (BS). To enhance performance of distance users, we need $N$ relay nodes. Regarding distance users, two destination $U_{1}, U_{2}$, one eavesdropper $E$ are considered their performance. Through the paper, $h_{u}$ is denoted as channel for node $u$, and it follows Rayleigh fading model with channel gain $\lambda_{u}$. It is noted that $P_{S}$ is transmit power at BS and it is limited under constraint with the primary network which contains primary destination $P_{D}$. The interference channel from BS to the primary network is $h_{S P} . h_{R_{n}}$ is the channel between $B S$ and $R$. $h_{R_{n}}$ is denoted as the channel between relay and $U_{i}$.

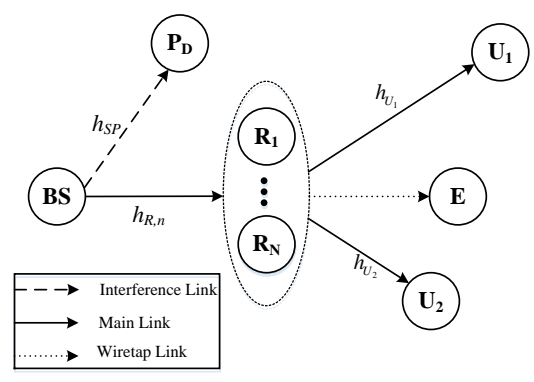

Figure 1. Secure CR-NOMA system

In CR-NOMA, the transmit power at the BS is constrained by (1)

$$
P_{S} \leq \min \left(\frac{I}{\left|h_{S P}\right|^{2}}, \bar{P}_{S}\right)
$$

in which, we call $\bar{P}_{S}$ and $I$ as maximum average transmit power at the BS and interference temperature constraint (ITC) at primary destination $P_{D}$, respectively. At the first hop transmission, the $n$-th node among $N$ relay node, the received signal can be formulated by (2)

$$
y_{R}=h_{R_{n}}\left(\sqrt{a_{1} P_{S}} x_{1}+\sqrt{a_{2} P_{S}} x_{2}\right)+n_{R_{n}}
$$

where the AWGN noise terms at $R$ is $n_{R_{n}}$. Regarding the relay is used to forward signal, the criteria to select relay with its index, i.e. $n^{*}$ is formulated related the best channel

$$
n^{*}=\arg \underbrace{\max }_{1,2, \ldots, N}\left|h_{R_{n}}\right|^{2}
$$

To decode signal $x_{1}$ and $x_{2}$ at $R$, the signal-to-interference-plus-noise ratio (SINR) is given by (4).

$$
S N R_{R}^{1}=\frac{a_{1} \rho_{S}\left|h_{R_{n}}\right|^{2}}{a_{2} \rho_{S}\left|h_{R_{n}}\right|^{2}+1}
$$

where $\rho_{S}=\frac{P_{S}}{\sigma^{2}}$ is the transmit signal to noise ratio (SNR) at the BS. At relay relying SIC, it is necessary to examine the received SNR at $R$ to detect message $x_{2}$ as (5).

$$
S N R_{R}^{2}=a_{2} \rho_{S}\left|h_{R n}\right|^{2}
$$

In the next phase, the link netween selected relay $R_{n}$ and $U_{i}, i=1,2$ is required to proceed signal $\sqrt{a_{1} P_{R}} \bar{x}_{1}+\sqrt{a_{2} P_{R}} \bar{x}_{2} . P_{R}$ is called as the transmit power at $R$. The received signal $U_{i}$ is expressed by (6)

$$
y_{U_{i}}=h_{U_{i}}\left(\sqrt{a_{1} P_{R}} \bar{x}_{1}+\sqrt{a_{2} P_{R}} \bar{x}_{2}\right)+n_{U_{i}} \quad, \forall i \in\{1,2\}
$$

in which the AWGN noise terms is $n_{U_{i}}$ measured at $U_{i}$. Further, principle of NOMA applied to $U_{i}$ with higher power factor, it can detect $\bar{x}_{1}$ by considering $\bar{x}_{2}$ as a background noise with (7) 


$$
S N R_{U_{1}}=\frac{a_{1} \rho_{R}\left|h_{U_{1}}\right|^{2}}{a_{2} \rho_{R}\left|h_{U_{1}}\right|^{2}+1}
$$

where $\rho_{R}=\frac{P_{R}}{\sigma^{2}}$ is the transmit SNR at $R$. To continue detecting procedure, $U_{2}$ needs SIC to decode $\bar{x}_{1}$ while considering its own data $\bar{x}_{2}$ as a noise. The SINR is written as (8).

$$
S N R_{U_{2}, x_{1}}=\frac{a_{1} \rho_{R}\left|h_{U_{2}}\right|^{2}}{a_{2} \rho_{R}\left|h_{U_{2}}\right|^{2}+1}
$$

In this regard, $U_{2}$ detects its own signal and the corresponding SNR is given as (9).

$$
S N R_{U_{2}, x_{2}}=a_{2} \rho_{R}\left|h_{U_{2}}\right|^{2}
$$

Unfortunately, eavesdropper steals information from the selected relay, the received signal at $E$ is given as (10)

$$
y_{E}=h_{E}\left(\sqrt{a_{1} P_{E}} \bar{x}_{1}+\sqrt{a_{2} P_{E}} \bar{x}_{1}\right)+n_{E}
$$

where $n_{E}$ is the AWGN noise terms at $E$. The channel between Relay and $\mathrm{E}$ is $h_{E}$. Then, SNR at $\mathrm{E}$ is given as

$$
S N R_{E i}=a_{i} \rho_{E}\left|h_{E}\right|^{2} \quad, i \in\{1,2\}
$$

where $\rho_{E}=\frac{P_{E}}{\sigma^{2}}$ is transmit SNR at $E$. The secrecy capacity for $U_{1}, U_{2}$ are computed respectively as

$$
\begin{gathered}
C_{1}=\left[\frac{1}{2} \log _{2}\left(\frac{1+\min \left(S N R_{R}^{1}, S N R_{U_{1}}\right)}{1+S N R_{E_{1}}}\right)\right]^{+}, \\
C_{2}=\left[\frac{1}{2} \log _{2}\left(\frac{1+\min \left(S N R_{R}^{2}, S N R_{U_{2}, x_{2}}\right)}{1+S N R_{E_{2}}}\right)\right]^{+},
\end{gathered}
$$

where $[x]^{+}=\max [x, 0]$.

\section{SPSC ANALYSIS}

3.1. SPSC computation at $U_{1}$

We first using decode-and-forward scheme at relay node and consider SPSC performance of the first user $U_{1}$ as (14).

$$
\begin{aligned}
G_{1}= & \operatorname{Pr}\left(C_{1}>0\right) \\
= & \operatorname{Pr}\left(\min \left(S N R_{R}^{1}, S N R_{U_{1}}\right)>S N R_{E 1}\right) \\
= & \underbrace{\operatorname{Pr}\left(\left|h_{R_{n^{*}}}\right|^{2}>\frac{\rho_{E}\left|h_{E}\right|^{2}}{\bar{\rho}_{S}\left(1-\rho_{E} a_{2}\left|h_{E}\right|^{2}\right)}, \bar{\rho}_{S}<\frac{\rho_{I}}{\left|h_{S P}\right|^{2}}\right)}_{G_{1,1}} \\
& +\underbrace{\operatorname{Pr}\left(\left|h_{R_{n^{*}}}\right|^{2}>\frac{\rho_{E}\left|h_{E}\right|^{2}\left|h_{S P}\right|^{2}}{\rho_{I}\left(1-a_{2} \rho_{E}\left|h_{E}\right|^{2}\right)}, \bar{\rho}_{S}>\frac{\rho_{I}}{\left|h_{S P}\right|^{2}}\right)}_{G_{G_{1,3}}}] \\
& \times \underbrace{\operatorname{Pr}\left(\left|h_{U_{1}}\right|^{2}>\frac{\rho_{E}\left|h_{E}\right|^{2}}{\rho_{R}\left(1-a_{2} \rho_{E}\left|h_{E}\right|^{2}\right)}\right)}_{G_{1,2}}
\end{aligned}
$$


We then compute each component $G_{1,1}, G_{1,2}, G_{1,3}$ as (15).

$$
\begin{aligned}
G_{1,1} & =\operatorname{Pr}\left(\left.\left|h_{\left.R_{n^{*}}\right|^{2}}>\frac{\rho_{E}\left|h_{E}\right|^{2}}{\bar{\rho}_{S}\left(1-\rho_{E} a_{2}\left|h_{E}\right|^{2}\right)},\right| h_{E}\right|^{2}<\eta, \bar{\rho}_{S}<\frac{\rho_{I}}{\left|h_{S P}\right|^{2}}\right) \\
& =\int_{0}^{\frac{\rho_{I}}{\bar{\rho}_{S}}} f_{\left|h_{S P}\right|^{2}}(x) \int_{0}^{\eta} f_{\left|h_{E}\right|^{2}}(y)\left[1-F_{\left|h_{R_{n}}\right|^{2}}\left(\frac{\rho_{E} y}{\bar{\rho}_{S}\left(1-\rho_{E} a_{2} y\right)}\right)\right] d x d y \\
& =\left(1-e^{-\frac{\rho_{I}}{\bar{\rho}_{S} \lambda_{S P}}}\right) \sum_{n=1}^{N}\left(\begin{array}{c}
N \\
n
\end{array}\right) \frac{(-1)^{n-1}}{\lambda_{E}} \int_{0}^{\eta} e^{-y\left(\frac{1}{\lambda_{E}}+\frac{n \rho_{E}}{\bar{\rho}_{S} \lambda_{S R}\left(1-a_{2} \rho_{E} y\right)}\right)} d y
\end{aligned}
$$

where $\eta=\frac{1}{a_{2} \rho_{E}}$.

The closed-form expression for $G_{1,1}$ is very difficult to achieve, then using the formula GaussianChebyshev quadrature, $G_{1,1}$ is given as (16)

$$
\begin{aligned}
G_{1,1} \approx & \left(1-e^{-\frac{\rho_{I}}{\bar{\rho}_{S} \lambda_{S P}}}\right) \sum_{n=1}^{N} \sum_{p=1}^{P}() \frac{\eta \pi(-1)^{n-1} \sqrt{1-\varsigma_{p}^{2}}}{2 P \lambda_{E}} \\
& \times e^{-\frac{\left(\varsigma_{p}+1\right) \eta}{2 \lambda_{E}}-\frac{\left(\eta \varsigma_{p}+\eta\right) \rho_{\rho_{E}}}{\bar{\rho}_{S} \lambda_{S R}\left[2-a_{2} \rho_{E}\left(\eta \varsigma_{p}+\eta\right)\right]}}
\end{aligned}
$$

where $\varsigma_{p}=\cos \left(\frac{2 p-1}{2 P} \pi\right)$.

Next, $G_{1,2}$ is calculated as (17).

$$
\begin{aligned}
G_{1,2}= & \operatorname{Pr}\left(\left|h_{R_{n^{*}}}\right|^{2}>\frac{\rho_{E}\left|h_{E}\right|^{2}\left|h_{S P}\right|^{2}}{\rho_{I}\left(1-a_{2} \rho_{E}\left|h_{E}\right|^{2}\right)},\left|h_{E}\right|^{2}<\eta, \bar{\rho}_{S}>\frac{\rho_{I}}{\left|h_{S P}\right|^{2}}\right) \\
= & \int_{0}^{\eta} f_{\left|h_{E}\right|^{2}}(x) \int_{\frac{\rho_{I}}{\bar{\rho}_{S}}}^{\infty} f_{\left|h_{S P}\right|^{2}}(y)\left[1-F_{\left|h_{R_{n}}\right|^{2}}\left(\frac{\rho_{E} x y}{\rho_{I}\left(1-a_{2} \rho_{E} x\right)}\right)\right] d x d y \\
= & \sum_{n=1}^{N}\left(\begin{array}{c}
N \\
n
\end{array}\right) \frac{(-1)^{n-1}}{\lambda_{S P} \lambda_{E}} \int_{0}^{\eta} e^{-\frac{x}{\lambda_{E}}} \int_{\frac{\rho_{I}}{\bar{\rho}_{S}}}^{\infty} e^{-y\left(\frac{1}{\lambda_{S P}}+\frac{n \rho_{E} x}{\rho_{I}\left(1-a_{2} \rho_{E}\right)^{x} \lambda_{S R}}\right)} d x d y \\
= & \sum_{n=1}^{N}\left(\begin{array}{c}
N \\
n
\end{array}\right) \frac{(-1)^{n-1}}{\lambda_{S P} \lambda_{E}} \int_{0}^{\eta} e^{-\frac{x}{\lambda_{E}}-\frac{\rho_{I}}{\bar{\rho}_{S}}}\left(\frac{1}{\lambda_{S P}}+\frac{n \rho_{E} x}{\rho_{I}\left(1-a_{2} \rho_{E} x\right) \lambda_{S R}}\right) \\
& \times\left(\frac{1}{\lambda_{S P}}+\frac{n \rho_{E} x}{\rho_{I}\left(1-a_{2} \rho_{E} x\right) \lambda_{S R}}\right)^{-1} d x
\end{aligned}
$$

Keep using the formula Gaussian-Chebyshev quadrature, $G_{1,2}$ is given by (18)

$$
G_{1,2} \approx \sum_{n=1}^{N} \sum_{v=1}^{V}(\quad) \frac{\eta \pi(-1)^{n-1} \sqrt{1-v_{v}^{2}}}{2 V \lambda_{S P} \lambda_{E} \Phi(w)} e^{-\frac{\left(v_{v}+1\right) \eta}{2 \lambda_{E}}-\frac{\rho_{I} \Phi\left(v_{v}\right)}{\bar{\rho}_{S}}}
$$

where $v_{v}=\cos \left(\frac{2 v-1}{2 V} \pi\right)$ and $\Phi(w)=\left(\frac{1}{\lambda_{S P}}+\frac{n \rho_{E}(\eta w+\eta)}{\left[2-a_{2} \rho_{E}(\eta w+\eta)\right] \rho_{I} \lambda_{S R}}\right)$. 
Next, $G_{1,3}$ is calculated as (19)

$$
\begin{aligned}
G_{1,3} & =\operatorname{Pr}\left(\left|h_{U_{1}}\right|^{2}>\frac{\rho_{E}\left|h_{E}\right|^{2}}{\rho_{R}\left(1-a_{2} \rho_{E}\left|h_{E}\right|^{2}\right)},\left|h_{E}\right|^{2}<\eta\right) \\
& =\int_{0}^{\eta} f_{\left|h_{E}\right|^{2}}(x)\left[1-F_{\left|h_{U_{1}}\right|^{2}}\left(\frac{\rho_{E} x}{\rho_{R}\left(1-a_{2} \rho_{E} x\right)}\right)\right] d x \\
& =\frac{1}{\lambda_{E}} \int_{0}^{\eta} e^{-x\left(\frac{1}{\lambda_{E}}+\frac{\rho_{E}}{\lambda_{U_{1}} \rho_{R}\left(1-a_{2} \rho_{E} x\right)}\right)} d x
\end{aligned}
$$

Keep using the formula Gaussian-Chebyshev quadrature, $G_{1,3}$ is given by (20)

$$
G_{1,3} \approx \frac{\pi \eta}{2 \lambda_{E} Q} \sum_{q=1}^{Q} \sqrt{1-\varsigma_{q}^{2}} e^{-\frac{\left(\varsigma_{q}+1\right) \eta}{2 \lambda_{E}}-\frac{\left(\varsigma_{q}+1\right) \rho_{E} \eta}{\lambda_{U_{1}} \rho_{R}\left[2-\left(\varsigma_{q}+1\right) a_{2} \rho_{E} \eta\right]}}
$$

where $\varsigma_{q}=\cos \left(\frac{2 q-1}{2 Q} \pi\right)$.

Substituting (20), (18) and (16) into (14), $G_{1}$ is given by (21)

$$
\begin{aligned}
G_{1}= & {\left[\left(1-e^{-\frac{\rho_{I}}{\rho_{S}{ }^{\lambda} S P}}\right) \sum_{n=1}^{N} \sum_{p=1}^{P}\left(\begin{array}{c}
N \\
n
\end{array}\right) \frac{\eta \pi(-1)^{n-1} \sqrt{1-\varsigma_{p}^{2}}}{2 P \lambda_{E}}\right.} \\
& \times e^{-\frac{\left(\varsigma_{p}+1\right) \eta}{2 \lambda_{E}}-\frac{\left(\eta \varsigma_{p}+\eta\right) n \rho_{E}}{\bar{\rho}_{S} \lambda_{S}\left[2-a_{2} \rho_{E}\left(\eta \varsigma_{p}+\eta\right)\right]}} \\
& \left.+\sum_{n=1}^{N} \sum_{v=1}^{V}\left(\begin{array}{c}
N \\
n
\end{array}\right) \frac{\eta \pi(-1)^{n-1} \sqrt{1-v_{v}^{2}}}{2 V \lambda_{S P} \lambda_{E} \Phi(w)} e^{-\frac{\left(v_{v}+1\right) \eta}{2 \lambda_{E}}-\frac{\rho_{I} \Phi\left(v_{v}\right)}{\bar{\rho}_{S}}}\right] \\
& \times \frac{\pi \eta}{2 \lambda_{E} Q} \sum_{q=1}^{Q} \sqrt{1-\varsigma_{q}^{2}} e^{-\frac{\left(\varsigma_{q}+1\right) \eta}{2 \lambda_{E}}-\frac{\left(\varsigma_{q}+1\right) \rho_{E} \eta}{\lambda_{U_{1} \rho_{R}\left[2-\left(\varsigma_{q}+1\right) a_{2} \rho_{E} \eta\right]}}}
\end{aligned}
$$

\subsection{SPSC computation at $U_{2}$}

In similar way, SPSC of user $U_{2}$ is expressed by (22)

$$
\begin{aligned}
G_{2}= & \operatorname{Pr}\left(C_{2}>0\right) \\
= & \operatorname{Pr}\left(\min \left(S N R_{R}^{2}, S N R_{U_{2}, x_{2}}\right)>S N R_{E 2}\right) \\
= & {[\underbrace{\operatorname{Pr}\left(\left|h_{R_{n^{*}}}\right|^{2}>\bar{a} a_{2} \rho_{E}\left|h_{E}\right|^{2},\left|h_{S P}\right|^{2}<\frac{\rho_{I}}{\bar{\rho}_{S}}\right)}_{G_{2,1}}} \\
& +\underbrace{\operatorname{Pr}\left(\left|h_{R_{n^{*}}}\right|^{2}>\tilde{a} a_{2} \rho_{E}\left|h_{E}\right|^{2}\left|h_{S P}\right|^{2},\left|h_{S P}\right|^{2}>\frac{\rho_{I}}{\bar{\rho}_{S}}\right)}_{G_{2,3}}] \\
& \times \underbrace{\operatorname{Pr}\left(\left|h_{U_{2}}\right|^{2}>\frac{\rho_{E}\left|h_{E}\right|^{2}}{\rho_{R}}\right)}_{G_{2,2}}
\end{aligned}
$$

where $\bar{a}=\frac{1}{a_{2} \bar{\rho}_{S}}$ and $\tilde{a}=\frac{1}{a_{2} \rho_{I}}$. These terms $G_{2,1}, G_{2,2}, G_{2,3}$ are respectively formulated by (23) and (24) 


$$
\begin{aligned}
& G_{2,1}=\operatorname{Pr}\left(\left|h_{R_{n^{*}}}\right|^{2}>\bar{a} a_{2} \rho_{E}\left|h_{E}\right|^{2},\left|h_{S P}\right|^{2}<\frac{\rho_{I}}{\bar{\rho}_{S}}\right) \\
& =\int_{0}^{\frac{\rho_{I}}{\bar{\rho} S}} f_{\left|h_{S P}\right|^{2}}(x) \int_{0}^{\infty} f_{\left|h_{E}\right|^{2}}(y)\left[1-F_{\left|h_{R_{n^{*}}}\right|^{2}}\left(\bar{a} a_{2} \rho_{E} y\right)\right] d x d y \\
& =\left(1-e^{-\frac{\rho_{I}}{\bar{\rho}_{S}{ }^{\lambda} S P}}\right) \sum_{n=1}^{N}\left(\begin{array}{l}
N \\
n
\end{array}\right) \frac{(-1)^{n-1}}{\lambda_{E}} \int_{0}^{\infty} e^{-y\left(\frac{1}{\lambda_{E}}+\frac{n \bar{a} a_{2} \rho_{E}}{\lambda_{S R}}\right)} d y \\
& =\left(1-e^{-\frac{\rho_{I}}{\bar{\rho}_{S} \lambda_{S P}}}\right) \sum_{n=1}^{N}\left(\begin{array}{l}
N \\
n
\end{array}\right) \frac{(-1)^{n-1} \lambda_{S R}}{\lambda_{S R}+n \lambda_{E} \bar{a} a_{2} \rho_{E}} \\
& G_{2,2}=\operatorname{Pr}\left(\left|h_{R_{n^{*}}}\right|^{2}>\tilde{a} a_{2} \rho_{E}\left|h_{E}\right|^{2}\left|h_{S P}\right|^{2},\left|h_{S P}\right|^{2}>\frac{\rho_{I}}{\bar{\rho}_{S}}\right) \\
& =\int_{0}^{\infty} f_{\left|h_{E}\right|^{2}}(x) \int_{\frac{\rho_{I}}{\bar{\rho}_{S}}}^{\infty} f_{\left|h_{S P}\right|^{2}}(y)\left[1-F_{\left|h_{R_{n}}\right|^{2}}\left(\tilde{a} a_{2} \rho_{E} x y\right)\right] d x d y \\
& =\sum_{n=1}^{N}\left(\begin{array}{l}
N \\
n
\end{array}\right) \frac{\lambda_{S R}(-1)^{n-1} e^{-\frac{\rho_{I}}{\bar{\rho}_{S} \lambda_{S P}}}}{n a_{2} \tilde{a} \rho_{E} \lambda_{E} \lambda_{S P}} \int_{0}^{\infty} \frac{e^{-\ell_{n} x}}{\varpi_{n}+x} d x
\end{aligned}
$$

where $\ell_{n}=\frac{1}{\lambda_{E}}+\frac{n \tilde{a} a_{2} \rho_{E}}{\lambda_{S R}}$ and $\varpi_{n}=\frac{\lambda_{S R}}{\lambda_{S P} n \tilde{a} a_{2} \rho_{E}}$.

We are using [[25], 8.211.1], $G_{2,2}$ is given by (25).

$$
G_{2,2}=\sum_{n=1}^{N}\left(\begin{array}{l}
N \\
n
\end{array}\right) \frac{\lambda_{S R}(-1)^{n} e^{-\frac{\rho_{I}}{\bar{\rho} S \lambda_{S P}}+\ell_{n} \varpi_{n}}}{n a_{2} \tilde{a} \rho_{E} \lambda_{E} \lambda_{S P}} E i\left(-\ell_{n} \varpi_{n}\right)
$$

Next, we are calculated $G_{2,3}$ as (26).

$$
\begin{aligned}
G_{2,3} & =\operatorname{Pr}\left(\left|h_{U_{2}}\right|^{2}>\frac{\rho_{E}\left|h_{E}\right|^{2}}{\rho_{R}}\right) \\
& =\int_{0}^{\infty} f_{\left|h_{E}\right|^{2}}(x)\left[1-F_{\left|h_{U_{2}}\right|^{2}}\left(\frac{\rho_{E} x}{\rho_{R}}\right)\right] d x \\
& =\frac{1}{\lambda_{E}} \int_{0}^{\infty} e^{-x\left(\frac{1}{\lambda_{E}}+\frac{\rho_{E}}{\rho_{R} \lambda_{U_{2}}}\right)} d x \\
& =\frac{\rho_{R} \lambda_{U_{2}}}{\rho_{R} \lambda_{U_{2}}+\lambda_{E} \rho_{E}}
\end{aligned}
$$

Substituting (26), (25) and (23) into (22), $G_{2}$ is given by (27).

$$
\begin{aligned}
G_{2}= & \sum_{n=1}^{N}\left(\begin{array}{l}
N \\
n
\end{array}\right) \frac{\rho_{R} \lambda_{U_{2}} \lambda_{S R}}{\rho_{R} \lambda_{U_{2}}+\lambda_{E} \rho_{E}}\left[\left(1-e^{-\frac{\rho_{I}}{\bar{\rho}_{S} \lambda_{S P}}}\right) \frac{(-1)^{n-1}}{\lambda_{S R}+n \lambda_{E} \bar{a} a_{2} \rho_{E}}\right. \\
& \left.+\frac{(-1)^{n} e^{-\frac{\rho_{I}}{\bar{\rho}_{S} \lambda_{S P}}+\ell_{n} \varpi_{n}}}{n a_{2} \tilde{a} \rho_{E} \lambda_{E} \lambda_{S P}} E i\left(-\ell_{n} \varpi_{n}\right)\right]
\end{aligned}
$$




\section{NUMERICAL RESULTS}

Our simulation parameters here are $a_{1}=0.7$ and $a_{2}=0.3 . N=2, R_{1}=R_{2}=1 . \rho_{I}=5 d B . \rho_{E}=$ $15 d B . \lambda_{S P}=0.1, \lambda_{S R}=1, \lambda_{R D 1}=0.9, \lambda_{R D 2}=0.6$ and $\lambda_{R E}=0.1 . Q=V=1000$.

Figure 2 depicts SPSC performance versus transmit SNR. We consider many cases related to CRNOMA for $\rho_{E}=5,10,15(d B)$. It is very high value of SPSC when increasing $\rho$ to $20(\mathrm{~dB})$ and greater. Signal $x_{2}$ provides better SPSC performance compared with signal $x_{2}$, and it can be explained that different power allocated to each signal. Comparing the simulation results with the analytical results, there are tight matching curves.

It can be seen how the number of the relay nodes make impacts on SPSC performance at Figure 3. Similar trend of SPSC can be reported in Figure 3, it means SPSC will be worse as increasing $\rho$ to 30 (dB). Figure 3 depicts SPSC curves is highest at $N=15$. When $\rho$ is greater than $25(\mathrm{~dB})$, SPSC curves meet saturation.

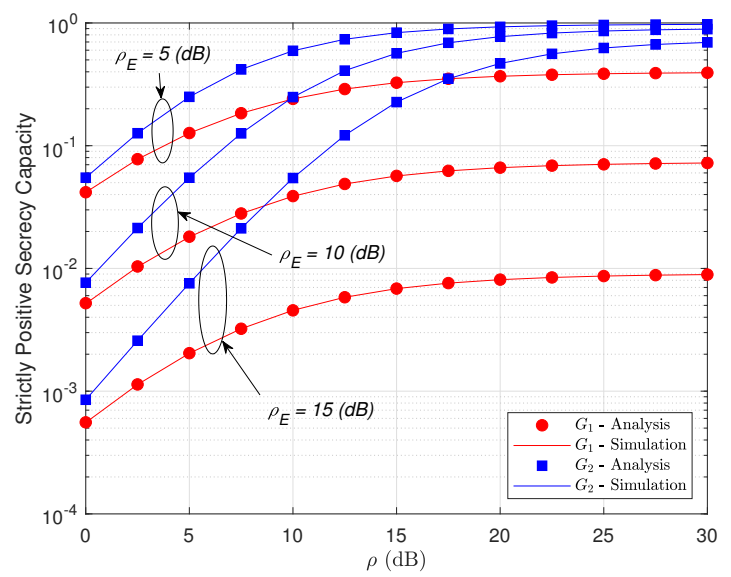

Figure 2.

SPSC versus transmit SNR at BS

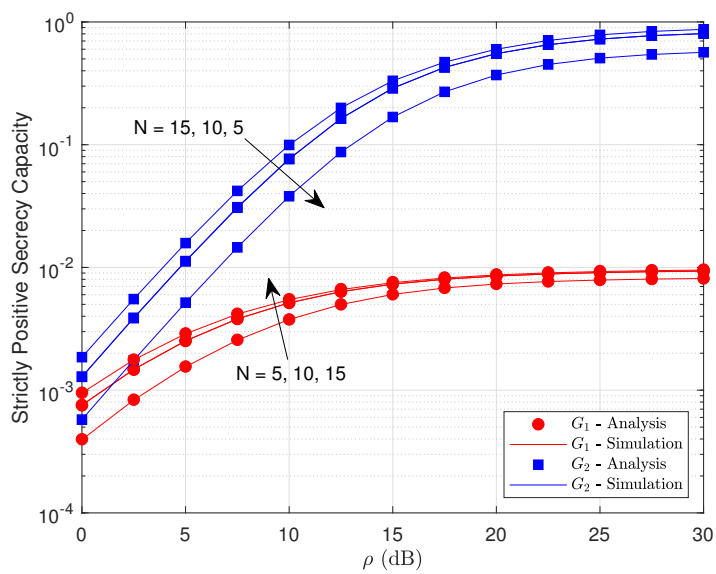

Figure 3. SPSC versus transmit SNR at BS with different number of relay nodes

\section{CONCLUSION}

The paper studied SPSC in cognitive radio newtwork using NOMA and relay selection. Secure performance is considered as existence of an eavesdropper and acceptable SPSC can be known. Moreover, the approximate expressions in term of SPSC are derived to exhibit performance of two destinations. The derivations and analysis results showed that the proposed relay selection in CR-NOMA can effectively enhance the secure performance.

\section{REFERENCES}

[1] Dinh-Thuan Do and M.-S. Van Nguyen, "Device-to-device transmission modes in NOMA network with and without Wireless Power Transfer," Computer Communications, Vol. 139, pp. 67-77, May 2019.

[2] Y. Liu, Z. Qin, M. Elkashlan, Y. Gao, and L. Hanzo, "Enhancing the Physical Layer Security of NonOrthogonal Multiple Access in LargeScale Networks," IEEE Trans. Wirel. Commun., vol. 16, no. 3, pp. 1656-1672, Mar. 2017.

[3] Y. Feng, Z. Yang, and S. Yan, "Non-orthogonal multiple access and artificial-noise aided secure transmission in FD relay networks," in Proc. IEEE GLOBECOM Wkshps, pp. 1-6, Dec. 2017.

[4] L. Lv, Z. Ding, Q. Ni, and J. Chen, "Secure MISO-NOMA transmission with artificial noise," IEEE Trans. on Vehic. Tech., vol. 67, no. 7, pp. 6700-6705, Jul. 2018.

[5] D.-T. Do, M. Vaezi, and T.-L. Nguyen, "Wireless Powered Cooperative Relaying using NOMA with Imperfect CSI," in Proc. of IEEE Globecom Workshops (GC Wkshps), Abu Dhabi, UAE, pp. 1-6, 2018.

[6] D.-T. Do and A.-T. Le, "NOMA based cognitive relaying: Transceiver hardware impairments, relay selection policies and outage performance comparison," Computer Communications, vol. 146, pp. 144-154, 2019. 
[7] D.-T. Do, A.-T. Le, and B.-M. Lee, "On Performance Analysis of Underlay Cognitive Radio-Aware Hybrid OMA/NOMA Networks with Imperfect CSI," Electronics, vol. 8, no. 7, p. 819, 2019.

[8] D.-T. Do, A.-T. Le, C.-B. Le, and B. M. Lee "On Exact Outage and Throughput Performance of Cognitive Radio based Non-Orthogonal Multiple Access Networks With and Without D2D Link," Sensors (Basel), vol. 19 , no. 15 , p. $3314,2019$.

[9] T.-L. Nguyen and Dinh-Thuan Do, "Exploiting Impacts of Intercell Interference on SWIPT-assisted Nonorthogonal Multiple Access ," Wireless Communications and Mobile Computing, vol. 2018, pp. 1-12, 2018.

[10] Dinh-Thuan Do, M.-S. Van Nguyen, T.-A. Hoang, and M. Voznak, "NOMA-Assisted Multiple Access Scheme for IoT Deployment: Relay Selection Model and Secrecy Performance Improvement," Sensors, vol. 19, no. 3, p. 736, 2019.

[11] F. Zhou, et al., "Robust AN-Aided beamforming and power splitting design for secure MISO cognitive radio with SWIPT," IEEE Trans. Wireless Commun., vol. 16, no. 4, pp. 2450-2464, Apr. 2017.

[12] H. Wang and X. Xia, "Enhancing wireless secrecy via cooperation: signal design and optmization," IEEE Commun. Magz., vol. 53, no. 12, pp. 47-53, 2015.

[13] E. Boshkovska, et al., "Robust resource allocation for MIMO wireless powered communication networks based on a non-linear EH model," IEEE Trans. Commun., vol. 65, no. 5, pp. 1984-1999, May 2017.

[14] E. Boshkovska, et al., "Secure SWIPT networks based on a non-linear energy harvesting model," in Proc. IEEE WCNC 2017, San Francisco, CA, USA, 2017.

[15] X. Chen, et al., "A survey on multiple-antenna techniques for physical layer security," IEEE Commun. Surveys Tuts., vol. 19, no. 2, pp. 1027-1053, 2017.

[16] Y. Liu, et al., "Phsical layer security for next generation wireless networks: Theories, tehcniques, and challenges," IEEE Commun. Surveys Tuts., vol. 19, no. 1, pp. 347-376, 2017

[17] Y. Sun, D. W. K. Ng, Z. Ding, and R. Schober, "Optimal joint power and subcarrier allocation for fullduplex multicarrier non-orthogonal multiple access systems," IEEE Trans. Commun., vol. 65, no. 3, pp. 1077-1091, 2017.

[18] H. Zhang, H. Xing, J. Cheng, A. Nallanathan, and V. C. M. Leung, "Secure resource allocation for OFDMA two-way relay wireless sensor networks without and with cooperative jamming," IEEE Trans. Ind. Informat., vol. 12, no. 5, pp. 1714-1725, Oct. 2016.

[19] Y. Wu, A. Khisti, C. Xiao, G. Caire, K.-K. Wong, and X. Gao, ”A survey of physical layer security techniques for 5g wireless networks and challenges ahead," IEEE J. Sel. Areas Commun., vol. 36, no. 4, pp. 679-695, Apr. 2018.

[20] Y. Deng, L. Wang, M. Elkashlan, A. Nallanathan, and R. K. Mallik, "Physical layer security in three-tier wireless sensor networks: A stochastic geometry approach,” IEEE Trans. Inf. Forensics Security, vol. 11, no. 6, pp. 1128-1138, Jun. 2016.

[21] F. Zhu and M. Yao, "Improving physical-layer security for CRNs using SINR-based cooperative beamforming," IEEE Trans. Veh. Technol., vol. 65, no. 3, pp. 1835-1841, Mar. 2016.

[22] A. Al-Nahari, G. Geraci, M. Al-Jamali, M. H. Ahmed, and N. Yang, "Beamforming with artificial noise for secure MISOME cognitive radio transmissions," IEEE Trans. Inf. Forensics Security, vol. 13, no. 8, pp. 1875-1889, Aug. 2018.

[23] P. Yan, Y. Zou, and J. Zhu, "Energy-aware multiuser scheduling for physical-layer security in energyharvesting underlay cognitive radio systems," IEEE Trans. Veh. Technol., vol. 67, no. 3, pp. 2084-2096, Mar. 2018.

[24] H. Lei, M. Xu, I. S. Ansari, G. Pan, K. A. Qaraqe, and M.-S. Alouini, ”On secure underlay MIMO cognitive radio networks with energy harvesting and transmit antenna selection," IEEE Trans. Green Commun. Netw., vol. 1, no. 2, pp. 192-203, Jun. 2017.

[25] I. S. Gradshteyn and I. M. Ryzhik, "Table of Integrals, Series and Products," 6th ed. New York, NY, USA: Academic Press, 2000. 